\title{
森林景观模型研究新进展及其应用
}

\author{
䍒为民 ${ }^{1,2}$,戴尔阜 3,4 , 贺红士 5,6
}

(1. Department of Biological and Health Sciences, Texas A\&M University, Kingsville, TX 78363, USA;

2. 中国科学院沈阳应用生态研究所, 沈阳 110016; 3. 中国科学院地理科学与资源研究所, 北京 100101 ;

4. 中国科学院陆地表层格局与模拟重点实验室, 北京 $100101 ; 5$. 东北师范大学,长春 130024;

6. School of Natural Resources, University of Missouri, Columbia MO 65211, USA)

\begin{abstract}
摘 要: 森林景观模型(forest landscape models)是基于森林动态机制和干扰作用在景观尺度上模拟和预测森林时空 变化特征的计算机模型。该类模型越来越多地用于森林规划、经营管理、生态资源保护与恢复及全球气候变化研 究。本文通过对大量文献资料的整理, 对森林景观模型的概念、尺度、类型、方法、应用和最新研究进展进行了综 述。随着计算机、地理信息系统、遥感等技术的迅猛发展,森林景观模型将会越来越多地与地理信息系统、规划经 营管理决策等紧密结合,未来将向服务性决策模型方向发展。
\end{abstract}

关 键 词: 森林景观变化; 景观模型; 时空尺度; 森林经营管理;资源保护与恢复; 决策性模型

1 引言

景观模型(landscape models) 是近 30 年来发展 起来的在景观尺度上模拟生态系统时空变化的数 学模型,并在计算机上加以体现和试验的技术; 为 现代景观生态学研究热点之一, 也是支撑景观生态 学定量化发展的重要技术手段(Baker, 1989; 邵国 凡, 1989; 奚为民等, 1992; He et al, 2002; Garman, 2004; Mladenoff, 2004; He, 2008; Xi et al, 2009)。景 观尺度是景观生态学研究的主要尺度, 基本对应中 尺度空间范围(从几十平方公里到几百平方公里)和 时间跨度(从几十年到几百年)。作为一种跨学科的 综合性技术手段, 景观模型是景观生态学、数量生 态学、现代计算机技术、遥感技术和地理信息系统 (Geographic Information Systems, GIS) 技术相结合 的产物。与其他尺度的生态模型相比, 景观模型具 有其自身的优势。例如, 与样地尺度上的植物生长 模型相比, 景观模型可以模拟区域生态系统的动态
变化并更能反映区域整体特征; 而与全球尺度的动 态植被模型相比, 景观模型不仅同样考虑了与大气 系统的交互作用,更能关注干扰因子对区域生态系 统的影响。景观模型经历了不同发展阶段, 早期的 模型侧重于对自然生态系统变化和自然干扰因子 (野火、风灾和虫灾等)相互作用的模拟,但随着景观 生态学研究领域的拓宽和景观模拟技术的发展, 景 观模拟研究逐渐包括了人文经济因素和社会变化 对自然生态系统的影响, 以及自然生态系统反馈和 社会发展效应等。景观模拟对提高生态系统长期 变化规律性的认识,特别是对全球气候变化大格局 下景观尺度上保护生物多样性和调控人类活动至 关重要(Turner, 1987; Shugart et al, 1992; 邵国凡等, 1996; Dale, 2003; He et al, 2005; Scheller et al, 2005; He, 2008; Xi et al, 2009)。

森林景观模拟(forest landscape modeling)是景 观模拟的重要组成部分之一。森林生态系统是全 球生态系统的重要组成部分, 作为复杂的异质等级

收稿日期: 2015-03; 修订日期: 2015-09。

基金项目: 国家自然科学基金项目(31370483,31300507,41371199,31300404); 中国科学院王宽诚教育基金项目 [Foundation: National Natural Science Foundation of China, No.31370483, No.31300507, No.41371199, No.31300404; K.C.Wong Education Foundation]。

作者简介: 奚为民(1963-), 男,博士, 研究员,主要从事森林生态系统、景观过程模拟、全球变化效应、生态系统管理和可持续发 展研究,E-mail: weiminxi305@gmail.com。

引用格式: 奚为民, 戴尔阜, 贺红士. 2016. 森林景观模型研究新进展及其应用[J]. 地理科学进展, 35(1): 35-46. [Xi W M, Dai E F, He H S. 2016. Advances in forest landscape modeling: Current research and applications[J]. Progress in Geography, 35(1): 35-46.]. DOI: $10.18306 /$ dlkxjz.2016.01.005 
系统, 森林生态系统的组成、结构和功能处于不断 的变化之中。其变化规律和影响因子又因观测的 时空尺度的不同而异。森林景观模拟以森林生态 系统为模拟的核心主体, 在生态系统的非平衡理论 (non-equilibrium theory) 和等级结构理论 (hierarchical structure theory)的指导下, 以森林生态系统变化 的主要过程和格局量化成果为基础, 根据生态系统 演替(succession)和干扰(disturbance)学说, 运用先进 的计算机技术拟合手段, 定量化地揭示森林景观和 相邻生态系统中树种生物学特性、种内种间竞争关 系及与生态环境干扰因子的综合作用效应(synergistic effects)。由于森林景观模型能在景观尺度上 对森林树种的组成、结构(包括多度和生物量等)和 功能(包括碳氮循环和生态服务等)的变化趋势予以 定量描述和预测, 还可揭示主要树木种群的空间地 理区范围和分布边界的变化与其自然和人为的影 响因素关系, 因此广泛地应用于森林动态、景观和 土地变化研究以及资源管理和生态环境评价等诸 多领域 (Mladenoff et al, 1999; He, 2008; Xi et al, 2008)。

随着计算机技术和新兴软件工程技术的快速 发展和广泛应用, 森林景观模拟技术也相应地迅速 发展。在此基础上开发出来的森林景观模型也不 断完善, 其应用范围也从早期的基础研究拓宽到林 业应用。有关森林景观模拟理论研究、技术开发和 模型应用的出版物也不断涌现。在国际上目前已 有几本英文专著和杂志专刊出版, 还有若干篇英文 综述文章阐述森林景观模拟技术的发展过程、森林 景观模型的主要类型、研究现状及其在森林生态学 研究和林业管理中的应用, 并对目前国际上主要的 森林景观模型的特点和适用领域进行对比和介绍 (Scheller et al, 2005; He, 2008; Xi et al, 2009; Dai et $\mathrm{al}, 2015)$; 在国内, 郭晋平等探讨了森林景观模型进 展(郭晋平等, 2001)。中国科学院应用生态研究所 和东北林业大学等单位开展了森林景观模型开发 和应用性研究, 取得了有一定影响的成果(He et al, 2005; Wang et al, 2014)。但总的看, 国内有关森林 景观模型的文献较少, 基础理论研究和实践应用还 不够深人。本文以多年来在中国和北美地区开展 的森林景观模拟工作为基础, 综合国际上森林景观 模拟研究现状, 对近 10 年来森林景观模拟工作和研 究开发应用的新进展加以综述, 提出开展森林景观 模拟研究需要注意的问题和发展前景, 以期促进森 林景观模拟研究的发展和有效的应用。

\section{2 森林景观模型概述}

\section{1 森林景观模型的概念}

明确森林景观模型的概念和范畴对文献综述 十分必要。不同学者对森林景观模型的理解和界 定不尽相同。例如, Mladenoff等定义森林景观模 型为在较宏观的时空尺度(spatio-temporal scale)上 模拟森林景观变化的各种计算机模型(Mladenoff et al, 1999); Scheller 等则定义森林景观模型为反映景 观变化的计算机程序集或软件包(Scheller, Mladenoff et al, 2007)。He 提出的较严格的森林景观模型 定义为: 可以预测模拟对象(simulation entity)的空 间特征(如分布、形状、丰度等)变化的模型; 同时提 出森林景观模型应至少能够以空间交互的方式 (spatially interactive manner)模拟周期性发生的空间 过程的时空特征(He, 2008)。Xi 等认为, 森林景观 模型是基于森林动态机制和干扰作用, 在景观尺度 上模拟和预测森林时空变化趋势的计算机模型, 其 研究对象包含森林景观中多种生态过程与空间格 局的交互作用(interactive interaction)和干扰(如生长 与演替过程, 碳氮等矿物质循环、水循环、森林火 灾、虫害与病害发展机制、气候变化及其影响机制 等)对动植物生境的影响(Xi et al, 2009)。

\section{2 森林景观模型的基础理论和建模方法}

不断发展中的演替、干扰与生态系统的非平衡 假说构成了生态模型开发的基础。生态学理论经 历了从自然界是平衡的、生态演替具有顶级群落的 观念向生态系统是一个动态的空间异质体的思想 转变(Wu et al, 1995; Perry et al, 2002)。这种观念上 的变化是森林景观模型建模的生态学基础(Perry et al, 2006)。森林景观模型的发展是过去二三十年中 生物地理学者和生态学家对森林景观演替、多因子干 扰和生态系统非平衡现象不断抽象并量化的过程。

早期的森林动态空间模型将元胞自动机(cellular automata)方法和森林动态原理相结合而发展起 来的(Jeltsch et al, 2002)。该方法已经日益复杂化, 并广泛用于生态学问题的研究 (Hargrove et al, 2000; Perry et al, 2002; Li et al, 2008)。等级分类理 论(hierarchy theory)是关于复杂系统结构、功能和动 态的理论, 是建立多尺度复杂系统模型的理论基础 (Costanza et al, 2004)。该理论以系统论、信息论、现 代哲学和数学等相关理论为基础, 为跨尺度的推绎 研究提供了一种系统的科学思路。空间动态集成、 尺度推绎等方法论的发展使建模过程更为客观有 
序, 促进了森林景观模型的发展。尺度推绎是指利 用某一尺度上所获得的信息和知识来推测其他尺 度上的现象。当从一个尺度推绎到另外一个尺度， 不同种类信息的保存与丢失也是跨尺度信息传递 研究的关键。尺度推绎有助于空间现象在不同尺 度上的综合(Costanza et al, 2004)。对空间推绎景观 模型而言, 尺度推绎也是融合和处理空间数据和社 会数据的关键。

\section{3 森林景观模型的尺度}

尺度(scale) 是景观生态学的重要特征, 也是森 林景观建模理论研究和模型应用的重要内容。森 林景观模型的模拟对象是各种森林景观 (forest landscape)。空间型森林景观模型通常用于模拟几 十年甚至几百年的景观变迁趋势。一般来说, 森林 景观模型所模拟的时间尺度(temporal scale)从几十 年到几百年(一般约 50 500 a); 空间尺度 (spatial scale)大致为 100 10000 km²。森林景观模型的尺度 是指模型所模拟研究的适宜时空幅度。同时也包 括模型的空间粒度大小 (spatial grain size)、时间步 长 (time step) 和复杂度 (degree of complexity) (Costanza et al, 2004)。时间解析度与模型的重复次 数有关, 是指模拟时的最小时间步长。由于每个景 观模型通常包含多个模拟目标过程, 而每个过程又 有其自身的时空尺度, 因而景观模型可以同时具有 多个时空解析度。值得注意的是, 当重复次数一定 时, 单一的时空解析度无法同时反映各个模拟 目标。

选择适宜的模型尺度是森林景观模型开发和 应用时所面临的关键问题(Xi et al, 2008)。由于空 间格局的复杂性, 目前没有一种单一模型可以清晰 地表达所有尺度的全部特征或信息。一方面, 景观 生态学家需要在适当的尺度上理解具体的生态过 程; 另一方面, 林地拥有者和管理人员又必须在较 大尺度上制定宏观管理政策(Rastetter et al, 2003)。 森林景观模型的开发者必须综合考虑以上两种因 素, 并针对具体的景观模拟对象和已有的计算机软 硬件条件做出相应的折中选择, 其中, LANDIS PRO 就将立地尺度与景观尺度有机结合, 并成功地 应用于区域森林景观的研究中(Wang et al, 2014)。 综上, 在模型模拟中如何更有针对性地确定 “最佳” 或比较合理的森林景观模型的尺度, 以及与此相关 的重要理论问题, 如景观尺度推绎(scaling) 和跨尺 度景观模拟(across-scale landscape modeling), 仍有 待进一步探讨。

\section{4 森林景观模型的类型}

与一般生态学模型不同, 森林景观模型中增加 了空间维度,即同时包含了时间和空间两个方面的 动态变化和交互作用。森林景观模型一般可分为 随机景观模型和过程景观模型。随机模型是由马 尔可夫过程理论发展而来, 它是基于转移概率将空 间信息与概率分布相结合的理论方法。当景观变 化的机理和多因子相互作用的联合效应尚不清楚 时, 景观生态学家常用随机模型来模拟自然因子和 人类活动对景观结构的影响。过程景观模型通过 建立尽可能真实的计算机模型来模拟景观的时空 交互过程。由于这种模型比较深人地研究组成景 观和生态系统的空间结构, 所以又被称作真实结构 模型。随着人工智能的理论和方法在生态学中的 应用,基于规则的景观模型将会得到进一步的完善 和应用, 成为解决复杂区域性资源与景观生态系统 管理问题的有效工具。

纵观森林景观模型发展, 不同学者对模型的分 类标准有着不同的理解。Horn 等将模拟景观变化 的生态模型分为两类: 分析模型和模拟模型(Horn et al, 1989)。分析模型是基于森林动态机制的分析 模型而建立的数学分析方程组, 侧重长期综合生态 系统动态分析(Perry et al, 2006), 因而又称作战略模 型(Verboom et al, 2005)或通用模型(Bolliger et al, 2005), 用于长期景观规划(Scheller, Mladenoff et al, 2007)。模拟模型通常运用更多的物理原理和非线 性直观方程组, 更注重所模拟系统的细节(Horn et al, 1989)。目前多数模拟模型都引人反复运行模型 结合统计分析方法, 称作战术模型(Verboom et al, 2005), 用于短期景观规划(Scheller, Mladenoff et al, 2007)。Perry 等根据模型是否模拟森林植被的动态 机制, 又将模拟模型细分为空间解译景观模型和林 窗动态模型(Perry et al, 2006)。空间解译景观模型 (Spatial explicit models) 是一种模拟和研究景观动 态机制的工具, 并 “体现了景观生态方法的精髓” (Baker, 1989)。这类模型假定景观空间组成结构随 着时间而变化,并且这些动态变化规律可通过特定 的数学关系来表示,一般适用于较大的时空尺度景 观问题。Baker将景观模型分为 3 类: 整体景观模型 (whole landscape models)、分布景观模型(distributional landscape models) 和空间景观模型 (spatial landscape models) (Baker, 1989)。Perry 等(2008)根 据模型的用途将空间模型分为: 预测模型 (predictive models)和探究式模型(exploratory models)。前 
者包括实验一统计模型 (empirical-statistical models)、转换矩阵模型(transition models)和林窗模型, 主要用于预测系统的未来变化; 后者主要用于探究 模拟对象的时空变化过程和变因, 又称启发式模型 (Perry et al, 2008)。Scheller等从生态功能的角度将 森林景观模型划分为 8 个类型, 主要基于以下 3 个 生态过程: 空间交互作用(spatial reciprocal interaction)、群落动态(tree species community dynamics)和 生态系统过程(ecosystem process)。该分类强调模 型组内在的关系, 在很大程度上反映了北美景观生 态学者注重景观空间过程和生态系统研究的传统 (Scheller, Mladenoff et al, 2007)。He在此基础上进 一步提出了森林景观模型分类的定量标准, 首先根 据森林景观模型是否模拟空间过程区分大类;再使 用模型的时间解析度和模拟森林演替方法等标准 划分更具体的模型类型 $(\mathrm{He}, 2008)$; 该分类标准强 调了模型开发人员在设计模型时对模型的时间解 析度、空间过程的数量和森林演替方法的选择。

\section{5 森林景观模型的发展}

作为景观模型(landscape models)的一个重要组 成部分, 森林景观模型的发展反映了森林生态学和 景观生态学的发展和融合的过程(Mladenoff et al, 1999)。早期, 北美景观生态学家和林业经营者在 寻求 “最佳” 经营和生态双重效益的思想指导下, 具 有悠久的开发和应用森林模型的历史(Mladenoff et al, 1999)。早期的非空间森林景观模型主要包括马 尔可夫转换模型(Markov chain model)和生命 - 属 性模型(vital-attribute model)(Mladenoff et al, 1999); 此后, 基于树木个体生长的林窗模型(forest gap models) 和林分生长与收获模型 (forest growth and yield model)得到了较大的发展。Botkin 等开发了 第一个林窗模型 (JABOWA 模型) (Botkin et al, 1972)。Shugart 等在 JABOWA 的基础上开发了 FORET 模型(Shugart, 1984)。林窗模型对于理解类 似森林生态系统中的森林林分动态机制有很重要 的贡献(Shugart, 1984; Urban et al, 1992)。

20 世纪 80 年代, 随着卫星遥感影像(如 Landsat 卫星的 $30 \mathrm{~m}$ 分辨率的 TM 数据) 和 GIS 软件的应用, 进行大幅度的空间分析成为可能。在计算速度和 存储能力取得巨大突破的同时, 各类编程语言和软 件的涌现使得模型的开发、输人输出数据更为便 捷, 大大提高了模型多尺度模拟运算的能力。同时 数据源也趋于多样化, 如土地覆盖的遥感影像、大 尺度的土壤类型空间数据库、植被样地(如美国森
林清查和分析项目, Forest Inventory and Analysis National Program)和土地利用调查数据以及历史资 料等数据源不断充实。在这样的发展环境驱动下， 森林学家越来越重视大尺度的景观分析和景观管 理问题,使得空间型森林景观模型的研究得到了迅 速发展。这一时期的森林景观模型开发逐渐形成 了两个基本特点:第一,森林景观模型具有空间特 性(Scheller, Mladenoff et al, 2007)。对景观变化进 行模拟必须对所有的景观要素进行地理编码, 具有 初始形状、空间定位, 或运用 GIS 进行数据的输人、 存储和显示等;第二, 森林景观模型强调大尺度驱 动因子(driving factors), 即各种环境干扰等(Baker, 1989; Mladenoff et al,1999)。目前森林景观模拟对 干扰的模拟不仅限于在较大尺度上模拟自然干扰 过程及其综合效应, 而且模拟人类活动(如林业采 伐、土地利用等)对景观变化造成的影响已成为森 林景观模拟的重要方面(Gustafson et al, 2000)。20 世纪 90 年代以后,森林景观模型的参数设计、初始 数据和验证数据都较以前有了较大改善。这个时 期的森林景观模型在参数设计方面普遍采用了两 种方法:物理方法和经验方法。物理方法是利用数 学方程建立物理变量和最终的预测结果的关系。 经验方法则是使用从物理变量中归纳出的组合参 数来模拟相关的变化过程。同期, 模拟多尺度、多 过程的森林景观模型得到了较快的发展,出现了诸 多的森林景观模型, 如 : FORMOSAIC(Liu et al, 1998), DELTA(Mladenoff et al, 1999), LANDSIM (Mladenoff et al, 1999) 和 LANDIS(He et al, 2004; Shang et al, 2004, 2007)等。He认为这个时期的森 林景观模型具有了生态系统过程模型的特点: 它不 仅追踪个体林木的空间变化,而且运用综合的物理 方法模拟控制关键生态过程的物流和能流 $(\mathrm{He}$, 2008)。森林景观模型的应用也不再限于单片林 区, 而更多地用于多个林区。

进人 21 世纪以来, 随着遥感探测(Remote Sensing, RS)技术的进一步发展, 景观生态学家们能迅 速地获取具有时间序列的遥感图像。遥感影像和 相关的 GIS 数据可直接用作森林景观模型的初始 数据, 对整个目标区域进行逐像元模拟,大区域和 高效计算机的出现及计算机图形技术的发展使大 规模图象处理及复杂运算更为快捷。因此,利用遥 感图像和空间分析软件进行景观变化的模拟和预 测, 已成为国内外研究者争相采用的方法 (Xi et al, 2009; He et al, 2011)。 


\section{3 森林景观模型应用的新进展}

森林景观模型的应用是指模型对实际问题的 综合和对具体模型结果的解释,包括与同行和应用 领域的专家交流并进一步完善模型。尽管森林景 观模型目前仍主要是研究性工具, 但它作为一种方 法探讨,为森林景观空间分布与环境因子变化的关 系以及森林景观对气候变化的反应, 提供了一种新 思路和有效方法。近 10 年来, 森林景观模型越来越 多地用于研究森林规划、经营管理、资源保护、生态 恢复、全球气候变化研究, 并逐渐成为辅助长期林 业景观规划和资源管理决策的一种有效工具(Perry et al, 2008; Xi et al, 2008)。

森林景观模型已越来越多地用于研究和解决 实际林业问题(Mladenoff, 2004)。He将景观模型的 应用归纳为 3 个方面: 模型目标的时空模拟、模型目 标对于输人参数的敏感性和经营预想方案的分析 (He, 2008)。目前, 森林景观模型已应用于林业经 营管理(Costanza et al, 2004)、流域规划和管理、灾后 森林景观的恢复 (Xi et al, 2007, 2008; Orsi et al, 2011)、林地利用发展规划等(Aghnoum et al, 2014; Könnyü et al, 2014)，其应用领域还在逐步扩大(表 1)。

在北美地区, 森林景观模型主要用于森林生态 系统的动态变化研究。如 Berland 等利用LANDISII 模型模拟美国明尼苏达州森林长期的动态变化, 利用模拟实验来评估影响该区域变化的重要生态 因子(Berland et al, 2011)。Seidl 等开发的 iLand 模 型用于模拟美国俄勒冈州景观尺度上的森林动态 性, 突出环境驱动因子在生态系统动态过程中的作 用,结果检验了模型处理复杂过程下模拟景观尺度 森林生态系统动态的能力(Seidl et al, 2012)。森林 资源的监测与规划是北美森林景观模型应用的又 一主要领域。如 Feng 等将 CA 模型(ALFRESCO)和 植物毒理响应模型(TDFRM)相结合, 模拟监测植物 毒性和营养级串联效应对美国阿拉斯加中部森林 火烧及演替格局的影响(Feng et al, 2012)。Staus 等 应用生态系统管理决策支持模型(EMDs) 对研究区 生态现状进行评价, 为俄勒冈州西部森林提供森林 管理与规划的政策支持(Staus et al, 2010)。而在欧 洲地区, 森林景观模型主要应用于探究气候变化对 森林景观结构与功能的影响。如 Henne 等利用 LandClim 模型模拟了全新世阿尔卑斯山植被动态, 并评估了浅层土壤对景观尺度上植被动态的影响，
从而探究植被对气候变暖的响应变化(Henne, 2011)。Gustafson 等利用 LANDIS-II 模型模拟了全 球变化对西伯利亚中南部森林景观的影响,对比分 析了森林砍伐、昆虫爆发以及气候变化的影响差 异,他们认为该地区森林对全球的变化响应强烈, 全球变化将显著地改变该地区森林景观组成及生 物量(Gustafson et al, 2010)。

森林景观模型的其它应用还包括林火管理和 林业经营管理(Scheller, Domingo et al, 2007; Shang et al, 2007)。LANDIS 系列模型已广泛地应用于减 缓林火的可燃物管理模拟,为森林火灾管理提供了 有效的科学支持(Scheller et al, 2011; Loudermilk et al, 2014)。如 He 等(2004)和 Yang 等 $(2004,2007)$ 利 用 LANDIS 研究了人类活动导致的林火干扰和不 同森林采伐方式的效应。同时在这些研究的基础 上,利用 LANDIS PRO 模型对美国中部阔叶林的森 林组成和结构进行了研究, 通过采用立地过程与景 观过程相结合的模拟方式,成功地预测了该区域森 林演替轨迹和森林发展格局(Wang et al, 2014)。 Scheller等利用 LANDIS-II 模型研究了在气候变化 条件下美国北部阔叶林与林火干扰、森林采伐方式 和风灾的空间关系以及天然林火对森林景观的长 期影响(Scheller et al, 2004, 2011; Scheller, Domingo et al, 2007)。此外,其他的森林景观模型也在林火 管理及林业经营中发挥重要的作用。如 FARSITE 火灾扩散模型利用地形、可燃的枯枝以及天气与风 的空间信息,将地表火(surface fire)、树冠火(crown fire)、星散火(spotting fire)及火灾扩展速率(fire acceleration)等模型集成到一个二维火灾模型中。该 模型用于预测林火发生时的可能传播模式,还用于 预测可燃枯枝空间变化。模型在近几年应用广泛， 对林火管理者大有帮助(Wehmeyer, 2012; Jahdi et al, 2014)。SAFE FORESTS 模型 (Sessions et al, 1999)着重于火灾动态以及林木采伐,已用于对内 华达山脉林火、次生林和林木采伐的联合管理。 LINKNZ模型探索史前森林景观, 是 LINKAGES 模 型的一个扩展模型,已用于新西兰的森林管理(Hall et al, 2000, 2006)。

中国在森林演替动态模型, 特别是森林景观模 型的开发和应用研究起步相对较晚。但自 1990年 以来, 在森林建模方面也有了长足的发展。森林景 观模型的引进和应用研究已经起步,并在近几年以 较快的速度发展。比如中国生态学者利用 LANDIS 模型在东北林区进行了较深人的森林动态和林业 
表 1 目前主要森林景观模型的方法和特点、关键问题和应用范围

Tab.1 A list of forest landscape models: features, key research questions, and applications

\begin{tabular}{|c|c|c|c|c|c|c|}
\hline 参考文献 & 模型名称 & 方法和特点 & 关键问题与应用范围 & $\begin{array}{l}\text { 空间幅度 } \\
\text { 和解析度 }\end{array}$ & $\begin{array}{l}\text { 空间 } \\
\text { 交互 }\end{array}$ & $\begin{array}{l}\text { 动态 } \\
\text { 模型 }\end{array}$ \\
\hline $\begin{array}{l}\text { Andrews } \\
(1986)\end{array}$ & BEHAVE & $\begin{array}{l}\text { 火行为预测 和森林可 } \\
\text { 燃物模拟的耦合模型 }\end{array}$ & $\begin{array}{l}\text { 估算森林可燃物与野火扩散方式, 用于预测 } \\
\text { 林火的扩散行为并提供有效的林火管理决策 }\end{array}$ & 不详 & 否 & 否 \\
\hline $\begin{array}{l}\text { Baker } \\
(1992)\end{array}$ & DISPATCH & 运用GIS管理空间数据 & $\begin{array}{l}\text { 探讨不同干扰作用和气候变化对美国明尼苏 } \\
\text { 达州景观结构的效应 }\end{array}$ & $\begin{array}{l}4000 \mathrm{~km}^{2} ; \\
200 \mathrm{~m} \text { 栅格 }\end{array}$ & 是 & 否 \\
\hline $\begin{array}{l}\text { Bugmann } \\
(1996)\end{array}$ & ForClim & $\begin{array}{l}\text { 模块化模型结构;包括 } \\
\text { 较少的生态假设,具体 } \\
\text { 的土壤过程 }\end{array}$ & $\begin{array}{l}\text { 应用整合环境(ForClim-E), 植物 (ForClim-P) } \\
\text { 与土壤(ForClim-S)等模块模拟欧洲阿尔卑斯 } \\
\text { 山区森林结构的长期(约 } 1200 \text { 年)变化 }\end{array}$ & 不详 & 否 & 是 \\
\hline $\begin{array}{l}\text { Li et al } \\
(1997)\end{array}$ & ONFIRE & $\begin{array}{l}\text { 侧重林火特征(如林火风 } \\
\text { 险和林火发生机率等) }\end{array}$ & $\begin{array}{l}\text { 模拟加拿大安大略省北部林区不同林火干扰 } \\
\text { 情形对森林景观结构的长期影响 }\end{array}$ & $\begin{array}{l}100 \mathrm{~km}^{2} ; \\
0.01 \mathrm{~km} \text { 栅格 }\end{array}$ & 是 & 否 \\
\hline $\begin{array}{l}\text { Baskent } \\
(1997)\end{array}$ & LANDMAN & $\begin{array}{l}\text { 基于 GIS 的空间数据和 } \\
\text { 景观管理模型 }\end{array}$ & $\begin{array}{l}\text { 探讨不同的初始景观结构和采伐模式导致的 } \\
\text { 加拿大New Brunswick地区未来森林景观变化 }\end{array}$ & $\begin{array}{l}43 \mathrm{~km}^{2} \text {; } \\
\text { 解析度不详 }\end{array}$ & 是 & 是 \\
\hline $\begin{array}{l}\text { Liu et al } \\
(1998)\end{array}$ & $\begin{array}{l}\text { FORMOSA } \\
\text { IC }\end{array}$ & $\begin{array}{l}\text { 整合森林管理策略,有 } \\
\text { 机与无机环境因子的 } \\
\text { 森林生长, 建立与死亡 } \\
\text { 动态模型 }\end{array}$ & $\begin{array}{l}\text { 探讨小尺度热带森林景观空间变化过程与相 } \\
\text { 邻区域生态条件的交互关系 }\end{array}$ & $\begin{array}{l}5 \mathrm{~km}^{2} ; \\
10 \mathrm{~m} \text { 栅格 }\end{array}$ & 是 & 是 \\
\hline $\begin{array}{l}\text { Mladenoff } \\
\text { et al (1999) }\end{array}$ & LANDIS & $\begin{array}{l}\text { 基于 JABOWA-FORET } \\
\text { 林窗与 LANDSIM 整合 } \\
\text { 的栅格模型 }\end{array}$ & $\begin{array}{l}\text { 引入概率和空间交互方法模拟森林景观空间 } \\
\text { 变化过程(如演替、森林衰退等), 探讨森林景 } \\
\text { 观与干扰(如林火)的交互作用 }\end{array}$ & $10 \sim 10000 \mathrm{~km}^{2}$ & 是 & 是 \\
\hline $\begin{array}{l}\text { Sessions et } \\
\text { al (1999) }\end{array}$ & $\begin{array}{l}\text { SAFE } \\
\text { FORESTS }\end{array}$ & $\begin{array}{l}\text { 基于非线性回归和栅 } \\
\text { 格模型 }\end{array}$ & $\begin{array}{l}\text { 研究火灾动态以及采伐对于内华达山脉森林 } \\
\text { 景观变迁的影响,并应用于对林火、次生林和 } \\
\text { 林木采伐的管理 }\end{array}$ & $\begin{array}{l}120 \mathrm{~km}^{2} ; \\
10 \sim 25 \mathrm{~km} \text { 栅格 }\end{array}$ & 是 & 否 \\
\hline $\begin{array}{l}\text { Dale et al } \\
(1999)\end{array}$ & DELTA & $\begin{array}{l}\text { 基于土地利用 GIS 的空 } \\
\text { 间数据和生态系动态 } \\
\text { 过程的整合模型 }\end{array}$ & $\begin{array}{l}\text { 应用概率与空间动态模型探讨人为土地利用 } \\
\text { 政策对改变巴西亚马逊地区森林景观的影 } \\
\text { 响,并估算森林破坏的速率 }\end{array}$ & $\begin{array}{l}296 \mathrm{~km}^{2} ; \\
0.53 \mathrm{~km} \text { 栅格 }\end{array}$ & 是 & 是 \\
\hline $\begin{array}{l}\text { Roberts et } \\
\text { al (1999) }\end{array}$ & LANDISIM & $\begin{array}{l}\text { 采用种类属性/模糊系 } \\
\text { 统模拟方法;空间解译 } \\
\text { 性模型 }\end{array}$ & $\begin{array}{l}\text { 模拟美国犹他州国家森林内树种分布与树龄 } \\
\text { 结构于空间与时间上的变化过程 }\end{array}$ & $\begin{array}{l}142.5 \mathrm{~km}^{2} ; \\
\text { 解析度不详 }\end{array}$ & 是 & 是 \\
\hline $\begin{array}{l}\text { Wimberly et } \\
\text { al (2000) }\end{array}$ & LADS & $\begin{array}{l}\text { 基于树龄级统计特征 } \\
\text { 的景观模拟模型 }\end{array}$ & $\begin{array}{l}\text { 模拟美国俄勒冈州沿岸地区森林的历史变化 } \\
\text { 和林火对森林结构和树种组成的长期(约 } \\
3000 \text { 年)影响 }\end{array}$ & $\begin{array}{l}400 \sim 22500 \mathrm{~km}^{2} ; \\
1 \mathrm{~km} \text { 栅格 }\end{array}$ & 是 & 是 \\
\hline $\begin{array}{l}\text { Klenner et } \\
\text { al (2000) }\end{array}$ & $\begin{array}{l}\text { VDDT/ } \\
\text { TELSA }\end{array}$ & $\begin{array}{l}\text { 空间解译模型; 强调森 } \\
\text { 林景观变迁与干扰与 } \\
\text { 森林管理策略的关系 }\end{array}$ & $\begin{array}{l}\text { 研究加拿大英属哥伦比亚地区森林管理策略 } \\
\text { 与自然干扰作用对森林内生物栖息地发展的 } \\
\text { 影响 }\end{array}$ & $62966 \mathrm{~km}^{2}$ & 是 & 否 \\
\hline $\begin{array}{l}\text { Li et al } \\
(2000)\end{array}$ & $\begin{array}{l}\text { SEM- } \\
\text { LAND }\end{array}$ & $\begin{array}{l}\text { 空间解译模型; 侧重于 } \\
\text { 模拟林火前后森林植 } \\
\text { 被与景观变化 }\end{array}$ & $\begin{array}{l}\text { 模拟不同林火特征(林火面积,发生频率,周期 } \\
\text { 等)对加拿大中西部森林景观结构的效应 }\end{array}$ & $\begin{array}{l}74.32 \mathrm{~km}^{2} ; \\
0.01 \mathrm{~km} \text { 栅格 }\end{array}$ & 是 & 否 \\
\hline $\begin{array}{l}\text { Hargrove et } \\
\text { al (2000) }\end{array}$ & EMBYR & 基于 GIS 空间数据模型 & $\begin{array}{l}\text { 运用概率统计模型模拟大尺度林火,并探讨 } \\
\text { 林火对不同景观结构的影响 }\end{array}$ & $\begin{array}{l}625 \mathrm{~km}^{2} ; \\
50 \mathrm{~m} \text { 栅格 }\end{array}$ & 是 & 是 \\
\hline $\begin{array}{l}\text { Gustafson et } \\
\text { al (2000) }\end{array}$ & t HARVEST & $\begin{array}{l}\text { LANDIS 模型的采伐 } \\
\text { 模块 }\end{array}$ & $\begin{array}{l}\text { 模拟美国东南部美国密苏里州森林在不同采 } \\
\text { 伐方式下森林景观的变化 }\end{array}$ & $\begin{array}{l}8.36 \mathrm{~km}^{2} ; \\
30 \mathrm{~m} \text { 栅格 }\end{array}$ & 是 & 否 \\
\hline $\begin{array}{l}\text { Yemshanov } \\
\text { et al (2002) }\end{array}$ & BFOLDS & $\begin{array}{l}\text { 建立在时变马尔可夫链 } \\
\text { 方法上的矩阵转换模型 }\end{array}$ & $\begin{array}{l}\text { 研究与预测加拿大北部森林林种长期动态变 } \\
\text { 化趋势, 并探讨干扰在其中所起的作用 }\end{array}$ & $\begin{array}{l}3.7 \times 10^{4} \mathrm{~km}^{2} ; \\
0.01 \mathrm{~km} \text { 栅格 }\end{array}$ & 是 & 是 \\
\hline $\begin{array}{l}\text { Keane et al } \\
(2002)\end{array}$ & LANDSUM & 空间解译模拟模型 & $\begin{array}{l}\text { 模拟美国西北部不同景观尺度上的植被分布 } \\
\text { 随时间尺度变化 }\end{array}$ & $\begin{array}{l}25 \sim 5160 \mathrm{~km}^{2} ; \\
\text { 解析度不详 }\end{array}$ & 是 & 是 \\
\hline $\begin{array}{l}\text { Pennanen et } \\
\text { al (2004) }\end{array}$ & Q-LAND & $\begin{array}{l}\text { LANDIS 扩展模型 } ; \text { 模 } \\
\text { 型林分尺度和景观尺 } \\
\text { 度过程 }\end{array}$ & $\begin{array}{l}\text { 整合种子传播方式与林分树木体积, 模拟加 } \\
\text { 拿大魁北克地区北方针阔混交林长期(约 } \\
1500 \text { 年)演替与景观结构变化 }\end{array}$ & $\begin{array}{l}\text { 约 } 1 \mathrm{~km}^{2} ; \\
0.01 \sim 0.1 \mathrm{~km} \text { 栅 } \\
\text { 格 }\end{array}$ & 是 & 是 \\
\hline $\begin{array}{l}\text { Pausas } \\
(2006)\end{array}$ & FATELAND & $\begin{array}{l}\text { 整合景观特性, 干扰作 } \\
\text { 用与植物分布动态变 } \\
\text { 化的柵格模型 }\end{array}$ & 研究林火和景观模式对于群落结构的影响 & $\begin{array}{l}1 \mathrm{~km}^{2} ; \\
10 \mathrm{~m} \text { 栅格 }\end{array}$ & 是 & 是 \\
\hline $\begin{array}{l}\text { Scheller et } \\
\text { al (2007a) }\end{array}$ & LANDIS-II & $\begin{array}{l}\text { LANDIS 扩展和升级模 } \\
\text { 型;包括生物量模块 }\end{array}$ & 模拟森林演替与干扰交互作用的关系与过程 & $\begin{array}{l}10^{4} \mathrm{~km}^{2} ; \\
50 \mathrm{~m} \text { 栅格 }\end{array}$ & 是 & 是 \\
\hline $\begin{array}{l}\text { Seidl et al } \\
(2012)\end{array}$ & iLand & $\begin{array}{l}\text { 以立地为基础的森林 } \\
\text { 景观干扰模型 }\end{array}$ & 模拟景观尺度上森林生态系统的动态变化 & $100 \mathrm{~m}$ 栅格 & 是 & 是 \\
\hline $\begin{array}{l}\text { Wang et al } \\
(2014) \\
\end{array}$ & $\begin{array}{l}\text { LANDIS } \\
\text { PRO }\end{array}$ & 新一代 LANDIS 模型 & $\begin{array}{l}\text { 预测美国中部阔叶森林组成与结构变化, 整 } \\
\text { 合了立地与景观过程 }\end{array}$ & $90 \mathrm{~m}$ 栅格 & 是 & 是 \\
\hline
\end{tabular}


管理方面的应用性研究 (He et al, 2002)。胡远满等 应用 LANDIS 模型研究了采伐和无采伐预案下大 兴安岭呼中林区森林景观的长期变化, 定量评价采 伐对森林景观变化的影响(胡远满等, 2004), 为森林 经营管理者提供决策依据。He等应用 LANDIS 模 型对长白山国家自然保护区的森林景观进行了长 期预测(He et al, 2005)。目前中国森林景观模型的 应用研究主要在东北林区, 其重点主要结合中国国 情, 模拟森林景观生态系统的演替规律、植物群落 的干扰机制和森林资源的动态与管理。

\section{4 森林景观模型面临的挑战}

\section{1 森林景观模型的局限性}

对于生态学家和森林管理者而言, 越来越多的 可用森林景观模型为空间模拟提供了机遇。但是, 友好的交互界面并不能克服每个模型自身固有的 局限性, 如森林景观模型的局限性首先是由于人类 对森林生态过程和格局认识的不完整性。因为模 拟景观变化不仅要了解现状景观演化到未来景观 的过程, 更重要的是要搞清景观发生变化的原因 (Schumacher et al, 2004)。其次, 森林景观模型在结 果验证上仍存在循环论证问题( $\mathrm{He}, 2008)$ 。这是由 于森林景观模型验证所需的时间和空间上相对独 立的序列性数据一般无法获得。根据传统的方法, 结果验证需要特定时间和空间数据来验证模型的 预测结果, 如果阶段性结果验证有效, 则一般就认 为后续的预测结果有效。但是森林景观模型不可 能用传统的方法对所有时间序列的数据进行验证, 因为如果整个时间序列都可验证, 那么森林景观模 型也就失去意义了。再次, 当研究人员讨论导致模 拟结果的生物的或非生物因素时,这些因素事实上 正是建模时采用并希望得到的模拟结果。用于数 据分析和结果验证的方法和工具是森林景观模型 未来需要提升的一个重要方面。LANDISVIEW 软 件是这方面的一个有益尝试 (Xi et al, 2008; Birt et al, 2009)。

\section{2 森林景观模型面临的挑战}

目前森林景观模型研究和应用所面临的挑战 主要包括: (1)如何确定模型的代表性, 如何把握好 模型尺度的选择, 如何进行尺度推绎; (2)如何处理 好林业管理和人为活动的关系, 在森林景观模型中 较好地模拟社会经济因素的作用; (3)如何更客观地 检验和分析模型的有效性和不确定性; (4)如何避免
模型的错用和滥用。特别值得强调的是, 未来的空 间推绎景观模型需要更多地考虑受人为因素以及 社会经济因素的影响(如土地利用、土地覆盖变化、 全球气候变化等) (Mladenoff et al, 1999; Xi et al, 2008; He et al, 2011)。这将有助于更好地将森林景 观模型用于对现实社会的环境和资源问题的规划 和管理。

在对景观管理的设计和评估方面, Perry等认为 空间解译景观模型在未来的发展中将面临模型应 用、数据采集和方便移植等多重挑战(Perry et al, 2006)。一是模型的易用性和模型结果的可视化(visualization)影响着景观模型的发展和应用。若要提 高模型应用的效率, 模型开发者和使用者需要相互 协作, 以使研究目标明确、框架合理; 二是使用空间 解译景观模型时也面临空间数据方面的限制。空 间解译景观模型往往十分依赖空间数据, 确定模型 的参数 (parameterization) 的过程亦费时费力, 上述 因素都制约了模型的广泛应用(Jeltsch et al, 2002); 三是空间解译景观模型及其核心程序的再利用 (model reuse)仍是一个尚未解决好的技术问题。开 发新的空间解译景观模型是一个既耗时又十分困 难的过程,尽可能利用已有的程序资源将大大缩短 新模型的开发周期。目前应用于开发景观模型的 模块和程序移植方法已处于研发阶段。

在森林监测和规划方面, 空间解译景观模型的 开发还需探索在不同时空尺度下提取数据的最佳 方法。比如Urban运用跨尺度数据对水分平衡条件 下森林景观对气候条件变化的敏感性进行了研究, 他使用较详细的空间数据和设计良好的森林动态 模型, 改进了提取景观要素中对气候变化较为敏感 的环境因子的方法(Urban, 2000)。

\section{5 对森林景观模型研究的前景展望}

在现代陆地森林生态系统研究中, 景观模型已 成为一种必不可少的手段。开发和完善森林景观 模型是现代生态学家十分关注的研究领域。从目 前森林景观模型的发展来看, 未来森林景观模型主 要发展趋势有以下几点:

(1) 不同类型模型耦合。每个模型都有自身适 用的领域, 同时也有一定的局限性。Perry等就认为 空间推绎景观模型与林窗模型体现了研究景观变 化的两种不同方法, 但这两种模型各有利弊(Perry et al, 2006)。使用林窗模型模拟景观级别的过程变 
化(如对干扰因子的变化反应)时会遇到问题, 因为 林窗模型并不包括发生在较大空间尺度的干扰生 态过程, 而空间推绎景观模型却能更好地反映这类 大尺度变化。尽管景观模型对研究干扰和景观变 化之间的相互作用很有效, 但这类模型往往不包括 管理森林生态系统所需要的树种年龄级和树木个 体空间分布的准确信息。正如 $\mathrm{He}$ 等所强调的, 用 单一的模型模拟跨越较大时空尺度的复杂景观生 态系统是不现实的(He et al, 1999)。将景观模型与 全球气候模型以及其他过程模型联合起来, 将会实 现各个模型之间取长补短。

(2) 与地理信息系统技术的结合。现代计算机 和空间图象处理技术, 特别是 GIS 、GPS (Global Positioning System)和 RS 的发展和完善, 大大加速了 森林景观模型开发的进程。目前可利用卫星光谱 图象和数字高程模型(digital elevation model, DEM) 对大范围的植被进行识别分析和分类制图, 并对综 合动态模型的结果进行快速验证。GIS 能够便捷地 处理空间推绎景观模型所需的空间数据, 可把植被 的动态与瞬时的气候条件结合起来以研究全球气 候变化对植被的响应以及植被对气候的反馈作 用。森林景观模型常采用松散耦合的方法与 GIS 结合使用,其优势是简便灵活。空间推绎景观模型 与 GIS 技术在不同层次的结合, 使得景观模型在处 理空间信息和研究空间过程方面的能力大大增强 (Fedra, 1993; Nyerges, 1993)。建立空间信息系统， 使用 GIS 处理和分析遥感数据, 将 GIS 、GPS 和 RS 等技术融合到森林景观模型中, 是当今景观模型发 展的热点和未来的重要方向(Pennanen et al, 2002)。

(3) 向服务性决策模型发展。Walters 认为要提 供决策支持, 景观模型需要考虑以下 3 个方面: (1)景 观模型应能较好地甄别敏感性强的因素; (2)考虑选 用适用的模型; (3)开发和应用模型的投人(Walters, 1993)。 $\mathrm{He}$ (2008)提出, 为了满足森林管理和规划的 实际需要, 森林景观模型应摆脱现有的理论探究模 式,而应以战略性预测模型作为未来的发展方向。

目前, 森林景观模型已不仅仅是一种研究工 具, 正逐渐在较大时间和空间尺度上成为一种辅助 林业管理的工具(Perry et al, 2006)。从管理规划部 门的角度考虑, 定量地分析预测结果及模型的准确 度, 将空间过程的模拟与传统的统计分析方法结合 起来十分必要(Landsberg, 2003)。今后森林景观模 型的发展会更多地对空间属性的变化过程进行综 合模拟。模拟研究的重点在于参数化过程中 “最
佳” 空间变量的判别和诸多空间过程的交互作用。

针对上述发展趋势, 我们认为今后森林景观模 型的研究应加强以下六方面:

一是建模理论和方法的研究。Urban认为关于 现有的建模基本理论通常过于简化, 与真实景观内 的物种复杂性和自然界演化的多样性不太符合。 需要根据自然界演化的历史和复杂的多尺度空间 过程进一步完善建模理论(Urban, 2005)。

二是开放式设计和公开程序问题。模型需要 合理地对用户和研究者开放。开放式的模型应允 许用户使用它的模型模块,而这类模型应在投人使 用前经过严格的测试和评估。新模型应可直接使 用以往模型的某些模块和数据(Syphard et al, 2004; Scheller, Domingo, 2007)。应逐渐建立标准化模型 程序模块库, 使之能较快捷方便地应用于其他模型 (He et al, 2005)。

三是模型程序的再利用、互利用及模型标准 化。实现这一设想首先需要确定利用目标, 建立利 用规范,使之可以操作。标准化的基本方法是对现 有的模型(程序)进行分类集成, 建立程序库, 逐步实 现建模的标准化。

四是加强计算机和地理信息技术等在森林景 观模型中的应用, 提高预测的准确度和效率。在景 观模型中适当地运用 GIS 模块, 提高嵌有 GIS 模块 的模型的可运行性(Mladenoff et al, 1999), 并使用多 个计算机处理器实现生态过程的同步运算 $(\mathrm{He}$, 2008), 解决好同步模拟生态过程的技术问题。未 来的森林景观模型需要更快捷的数学算法, 特别要 解决由于采用矢量方法而带来的计算量加大的 问题。

五是模拟结果的三维可视化。三维数字高程 模型是森林景观立体特征分析的基础。将遥感图 像与 DEM 重叠起来, 用户可自由设置观察路线和 角度, 模拟空中飞行, 对研究区进行多方位观察。 与已往的二维可视化不同,三维模拟能在不同地理 环境下模拟森林景观动态, 表达更为丰富的信息。

六是模型的有效性检验和应用。目前的景观 模型一般利用假设检验方法来评估模型的有效 性。这一传统意义上验证模型的方法已不能满足 开放性复杂随机模型的要求(Rykiel, 1996; He et al, 1999)。Rykiel 认为制定详细的规范模型验证标准 十分必要。他提出模型的验证可分为操作验证、概 念验证和数据验证(Rykiel, 1996)。Perry 等认为面 向格局法对评估空间推绎景观模型十分有效(Perry 
et al, 2002)。其主导思想是评价景观模型时要更多 地发掘不同空间过程和格局之间的联系, 进而通过 建立有效的模型结构框架以评估和比较模型的可 靠性和适用性(Grimm et al, 2013)。

景观模型模拟景观要素的时空过程, 揭示景观 变化规律, 不仅能储存过去和现存的植被信息、干 扰和管理的状态, 而且能预测景观要素的变化趋 势, 有助于更有效地研究森林对各种干扰的反应和 对森林景观的管理。由于它较好地模拟了空间格 局的复杂过程, 较清晰地表达时空特征的数量信 息, 因而在理论研究和实际应用方面都具有良好的 发展前景。可以预见, 未来几十年中, 生态学家对 森林景观的格局和过程的认识会进一步深化, 森林 景观模型的技术开发方法会更综合化, 森林景观模 型的类型会根据研究问题的不同而更趋于多样化。

\section{参考文献(References)}

郭晋平, 肖扬. 2001. 森林景观模型研究进展 [M]/李承森. 植 物科学进展: 第四卷. 北京: 高等教育出版社; 海德堡: 施普林格出版社: 255-272. [Guo J P, Xiao Y. 2001. Senlin jingguan moxing yanjiu jinzhan[M]//Li C S. Advances in plant sciences: Vol. 4. Beijing, China: Higher Education Press; Heidelberg, Germany: Springer: 255-272.]

胡远满, 徐崇刚, 常禹, 等. 2004. 空间直观景观模型 LANDIS 在大兴安岭呼中林区的应用 [J]. 生态学报, 24(9): 18461856. [Hu Y M, Xu C G, Chang Y, et al. 2004. Application of spatially explicit landscape model (LANDIS): A case researches in Huzhong area, Mt. Daxing' anling[J]. Acta Ecologica Sinica, 24(9): 1846-1856.]

邵国凡. 1989. 当代森林动态的计算机模型述评 [J]. 生态学 杂志, 8(2): 34-37. [Shao G F. 1989. A review on contemporary computer models of forest dynamics[J]. Journal of Ecology, 8(2): 34-37.]

邵国凡, 赵士洞, 舒噶特. 1996. 森林动态模拟: 兼论红松林 的优化经营 [M]. 北京: 中国林业出版社. [Shao G F, Zhao S D, Shugart H H. 1996. Forest dynamics modeling: Preliminary explanations of optimizing management for Korean pine forests[M]. Beijing: China Forestry Publishing House.]

奚为民, 钟章成, 毕润成. 1992. 林窗植被研究进展 [J]. 西南 师范大学学报: 自然科学版, 17(2): 268-274. [Xi W M, Zhong Z C, Bi R C. 1992. Advance in research of forest gaps vegetation[J]. Journal of Southwest China Teachers University: Natural Science, 17(2): 268-274.]

Aghnoum M, Feghhi J, Makhdoum M, et al. 2014. Assessing the environmental impacts of forest management plan based on matrix and landscape degradation model[J]. Journal of Agricultural Science and Technology, 16(4):
841-850.

Andrews P L. 1986. BEHAVE: Fire behavior prediction and fuel modeling system: BURN subsystem, part $1[\mathrm{R} / \mathrm{OL}]$. 2015- 11- 6. http://digitalcommons.usu.edu/cgi/viewcontent.cgi?article $=1148 \&$ context=barkbeetles.

Baker W L. 1989. A review of models of landscape change[J]. Landscape Ecology, 2(2): 111-133.

Baker W L. 1992. The landscape ecology of large disturbances in the design and management of nature reserves[J]. Landscape Ecology, 7(3): 181-194.

Baskent E Z. 1997. Assessment of structural dynamics in forest landscape management[J]. Canadian Journal of Forest Research, 27(10): 1675-1684.

Berland A, Shuman B, Manson S M. 2011. Simulated importance of dispersal, disturbance, and landscape history in long-term ecosystem change in the big woods of Minnesota[J]. Ecosystems, 14(3): 398-414.

Birt A G, Xi W M, Coulson R N. 2009. LANDISVIEW: A visualization tool for landscape modelling[J]. Environmental Modelling \& Software, 24(11): 1339-1341.

Bolliger J, Lischke H, Green D G. 2005. Simulating the spatial and temporal dynamics of landscapes using generic and complex models[J]. Ecological Complexity, 2(2): 107-116.

Botkin D B, Bartley H A, Wallis J R. 1972. Some ecological consequences of a computer model of forest growth[J]. Journal of Ecology, 60(3): 849-872.

Bugmann H K M. 1996. A simplified forest model to study species composition along climate gradients[J]. Ecology, 77(7): 2055-2074.

Costanza R, Voinov A. 2004. Landscape simulation modeling: A spatially explicit dynamic approach[M]. New York: Springer.

Dai E F, Wu Z, Wang X F, et al. 2015. Progress and prospect of research on forest landscape model[J]. Journal of Geographical Sciences, 25(1): 113-128.

Dale V H. 2003. Ecological modeling for resource management[M]. New York: Springer.

Dale V H, Pearson S M. 1999. Modeling the driving factors and ecological consequences of deforestation in the Brazilian Amazon[M]//Mladenoff D J, Baker W L. Spatial modeling of forest landscape change: Approaches and applications. Cambridge, UK: Cambridge University Press: 256-276.

Fedra K. 1993. GIS and environmental modeling[M]//Goodchild M F, Parks B O, Steyaert L T. Environmental modeling with GIS. New York: Oxford University Press: 33-51.

Feng Z L, Alfaro- Murillo J A, DeAngelis D L, et al. 2012. Plant toxins and trophic cascades alter fire regime and succession on a boreal forest landscape[J]. Ecological Modelling, 244: 79-92.

Garman S L. 2004. Design and evaluation of a forest landscape change model for western Oregon[J]. Ecological Modelling, 175(4): 319-337. 
Grimm V, Railsback S F. 2013. Individual-based modeling and ecology[M]. New Jersey: Princeton University Press.

Gustafson E J, Shifley S R, Mladenoff D J, et al. 2000. Spatial simulation of forest succession and timber harvesting using LANDIS[J]. Canadian Journal of Forest Research, 30 (1): 32-43.

Gustafson E J, Shvidenko A Z, Sturtevant B R, et al. 2010. Predicting global change effects on forest biomass and composition in south-central Siberia[J]. Ecological Applications, 20(3): 700-715.

Hall G M J, Hollinger D Y. 2000. Simulating New Zealand forest dynamics with a generalized temperate forest gap model[J]. Ecological Applications, 10(1): 115-130.

Hall G M J, McGlone M S. 2006. Potential forest cover of New Zealand as determined by an ecosystem process model[J]. New Zealand Journal of Botany, 44(2): 211-232.

Hargrove W W, Gardner R H, Turner M G, et al. 2000. Simulating fire patterns in heterogeneous landscapes[J]. Ecological Modelling, 125(2-3): 243-263.

He H S. 2008. Forest landscape models: Definitions, characterization, and classification[J]. Forest Ecology and Management, 254(3): 484-498.

He H S, Hao Z Q, Mladenoff D J, et al. 2005. Simulating forest ecosystem response to climate warming incorporating spatial effects in north-eastern China[J]. Journal of Biogeography, 32(12): 2043-2056.

He H S, Larsen D R, Mladenoff D J. 2002. Exploring component- based approaches in forest landscape modeling $[\mathrm{J}]$. Environmental Modelling \& Software, 17(6): 519-529.

He H S, Mladenoff D J. 1999. Spatially explicit and stochastic simulation of forest-landscape fire disturbance and succession[J]. Ecology, 80(1): 81-99.

He H S, Shang B Z, Crow T R, et al. 2004. Simulating forest fuel and fire risk dynamics across landscapes- LANDIS fuel module design[J]. Ecological Modelling, 180(1): 135-151.

He H S, Yang J, Shifley S R, et al. 2011. Challenges of forest landscape modeling-Simulating large landscapes and validating results[J]. Landscape and Urban Planning, 100(4): 400-402.

Henne P D, Elkin C M, Reineking B, et al. 2011. Did soil development limit spruce (Picea abies) expansion in the Central Alps during the Holocene: Testing a palaeobotanical hypothesis with a dynamic landscape model[J]. Journal of Biogeography, 38(5): 933-949.

Horn H S, Shugart H H, Urban D L. 1989. Simulators as models of forest dynamics[M]//Roughgarden J, May R M, Levin S A. Perspectives in ecological theory. New Jersey: Princeton University Press: 256-267.

Jahdi R, Salis M, Darvishsefat A A, et al. 2014. Calibration of FARSITE fire area simulator in Iranian northern forests [J]. Natural Hazards and Earth System Sciences Discussions, 2(9): 6201-6240.
Jeltsch F, Moloney K A. 2002. Spatially explicit vegetation models: What have we learned[M]//Esser K, Lüttge U, Beyschlag W, et al. Progress in botany: Genetics, physiology, ecology. Berlin, Heidelberg: Springer, 63: 326-343.

Keane R E, Parsons R A, Hessburg P F. 2002. Estimating historical range and variation of landscape patch dynamics: Limitations of the simulation approach[J]. Ecological Modelling, 151(1): 29-49.

Klenner W, Kurz W, Beukema S. 2000. Habitat patterns in forested landscapes: Management practices and the uncertainty associated with natural disturbances[J]. Computers and Electronics in Agriculture, 27(1-3): 243-262.

Könnyü N, Tóth S F, McDill M E, et al. 2014. Temporal connectivity of mature patches in forest planning models[J]. Forest Science, 60(6): 1089-1099.

Landsberg J. 2003. Modelling forest ecosystems: State of the art, challenges, and future directions $[\mathrm{J}]$. Canadian Journal of Forest Research, 33(3): 385-397.

Li C, Flannigan M D, Corns I G W. 2000. Influence of potential climate change on forest landscape dynamics of westcentral Alberta[J]. Canadian Journal of Forest Research, 30(12): 1905-1912.

Li C, Hans H, Barclay H, et al. 2008. Comparison of spatially explicit forest landscape fire disturbance models[J]. Forest Ecology and Management, 254(3): 499-510.

Li C, Perera A H. 1997. ON-FIRE: A landscape model for simulating the fire regime of northwest Ontario[M]//Chen X, Dai X, Hu T. Ecological research and sustainable development. Beijing, China: China Environmental Science Press: 369-392.

Liu J G, Ashton P S. 1998. FORMOSAIC: An individualbased spatially explicit model for simulating forest dynamics in landscape mosaics[J]. Ecological Modelling, 106(2-3): 177-200.

Loudermilk E L, Stanton A, Scheller R M, et al. 2014. Effectiveness of fuel treatments for mitigating wildfire risk and sequestering forest carbon: A case study in the Lake Tahoe Basin[J]. Forest Ecology and Management, 323: 114125.

Mladenoff D J. 2004. LANDIS and forest landscape models [J]. Ecological Modelling, 180(1): 7-19.

Mladenoff D J, Baker W B. 1999. Spatial modeling of forest landscape change: Approaches and application[M]. Cambridge, UK: Cambridge University Press.

Nyerges T L. 1993. Understanding the scope of GIS: Its relationship to environmental modeling[M]//Goodchild M F, Parks B O, Steyaert L T. Environmental modeling with GIS. New York: Oxford University Press: 75-93.

Orsi F, Church R L, Geneletti D. 2011. Restoring forest landscapes for biodiversity conservation and rural livelihoods: A spatial optimisation model[J]. Environmental Modelling \& Software, 26(12): 1622-1638. 
Pausas J G. 2006. Simulating Mediterranean landscape pattern and vegetation dynamics under different fire regimes[J]. Plant Ecology, 187(2): 249-259.

Pennanen J, Greene D F, Fortin M J, et al. 2004. Spatially explicit simulation of long-term boreal forest landscape dynamics: Incorporating quantitative stand attributes[J]. Ecological Modelling, 180(1): 195-209.

Pennanen J, Kuuluvainen T. 2002. A spatial simulation approach to natural forest landscape dynamics in boreal Fennoscandia[J]. Forest Ecology and Management, 164(1-3): 157-175.

Perry G L W, Enright N J. 2002. Spatial modelling of landscape composition and pattern in a maquis- forest complex, Mont Do, New Caledonia[J]. Ecological Modelling, 152(2-3): 279-302.

Perry G L W, Enright N J. 2006. Spatial modelling of vegetation change in dynamic landscapes: A review of methods and applications[J]. Progress in Physical Geography, 30 (1): 47-72.

Perry G L W, Millington J D A. 2008. Spatial modelling of succession-disturbance dynamics in forest ecosystems: Concepts and examples[J]. Perspectives in Plant Ecology, Evolution and Systematics, 9(3-4): 191-210.

Rastetter E B, Aber J D, Peters D P C, et al. 2003. Using mechanistic models to scale ecological processes across space and time[J]. BioScience, 53(1): 68-76.

Roberts D W, Betz D W. 1999. Simulating landscape vegetation dynamics of Bryce Canyon National Park with the vital attributes/fuzzy systems model VAFS/LANDSIM[M]// Mladenoff D J, Baker W L. Spatial modeling of forest landscape change: Approaches and applications. Cambridge, UK: Cambridge University Press: 99-123.

Rykiel E J Jr. 1996. Testing ecological models: The meaning of validation[J]. Ecological Modelling, 90(3): 229-244.

Scheller R M, Domingo J B, Sturtevant B R, et al. 2007. Design, development, and application of LANDIS-II, a spatial landscape simulation model with flexible temporal and spatial resolution[J]. Ecological Modelling, 201(3-4): 409-419.

Scheller R M, Mladenoff D J. 2004. A forest growth and biomass module for a landscape simulation model, LANDIS: Design, validation, and application[J]. Ecological Modelling, 180(1): 211-229.

Scheller R M, Mladenoff D J. 2005. A spatially interactive simulation of climate change, harvesting, wind, and tree species migration and projected changes to forest composition and biomass in northern Wisconsin, USA[J]. Global Change Biology, 11(2): 307-321.

Scheller R M, Mladenoff D J. 2007. An ecological classification of forest landscape simulation models: Tools and strategies for understanding broad-scale forested ecosystems[J]. Landscape Ecology, 22(4): 491-505.

Scheller R M, Spencer W D, Rustigian-Romsos H, et al. 2011.
Using stochastic simulation to evaluate competing risks of wildfires and fuels management on an isolated forest carnivore[J]. Landscape Ecology, 26(10): 1491-1504.

Schumacher S, Bugmann H, Mladenoff D J. 2004. Improving the formulation of tree growth and succession in a spatially explicit landscape model[J]. Ecological Modelling, 180 (1): 175-194

Seidl R, Rammer W, Scheller R M, et al. 2012. An individualbased process model to simulate landscape-scale forest ecosystem dynamics[J]. Ecological Modelling, 231(1): 87-100.

Sessions J, Johnson K N, Franklin J F, et al. 1999. Achieving sustainable forest structures on fire- prone landscapes while pursuing multiple goals[M]//Mladenoff D J, Baker W L. Spatial modeling of forest landscape change: Approaches and applications. Cambridge, UK: Cambridge University Press: 210-253.

Shang Z B, He S H, Crow R T, et al. 2004. Fuel load reductions and fire risk in central hardwood forests of the United States: A spatial simulation study[J]. Ecological Modelling, 180(1): 89-102.

Shang Z B, He H S, Lytle D E, et al. 2007. Modeling the longterm effects of fire suppression on central hardwood forests in Missouri Ozarks, using LANDIS[J]. Forest Ecology and Management, 242(2-3): 776-790.

Shugart H H. 1984. A theory of forest dynamics: The ecological implications of forest succession models[M]. New York: Springer.

Shugart H H, Smith T M, Post W M. 1992. The potential for application of individual-based simulation models for assessing the effects of global change[J]. Annual Review of Ecology and Systematics, 23(1): 15-38.

Staus N L, Strittholt J R, DellaSala D A. 2010. Evaluating areas of high conservation value in Western Oregon with a decision-support model[J]. Conservation Biology, 24(3): 711-720.

Syphard A D, Franklin J. 2004. Spatial aggregation effects on the simulation of landscape pattern and ecological processes in southern California plant communities[J]. Ecological Modelling, 180(1): 21-40.

Turner M G. 1987. Spatial simulation of landscape changes in Georgia: A comparison of 3 transition models[J]. Landscape Ecology, 1(1): 29-36.

Urban D L. 2000. Using model analysis to design monitoring programs for landscape management and impact assessment[J]. Ecological Applications, 10(6): 1820-1832.

Urban D L. 2005. Modeling ecological processes across scales [J]. Ecology, 86(8): 1996-2006.

Urban D L, Shugart H H. 1992. Individual-based models of forest succession[M]//Glennlewin D C, Peet R K, Veblen $\mathrm{T}$ T. Plant succession: Theory and prediction. London: Chapman and Hall. 
Verboom J, Wamelink W. 2005. Spatial modeling in landscape ecology[M]/Wiens J A, Moss M R. Issues and perspectives in landscape ecology. Cambridge, UK: Cambridge University Press: 79-89.

Walters C J. 1993. Dynamic models and large scale field experiments in environmental impact assessment and management[J]. Australian Journal of Ecology, 18(1): 53-61.

Wang W J, He H S, Fraser J S, et al. 2014. LANDIS PRO: A landscape model that predicts forest composition and structure changes at regional scales[J]. Ecography, 37(3): 225-229.

Wehmeyer G. 2012. Simulations and analysis of a 2012 Kansas Wildland fire using FARSITE[D]. Texas: The University of Texas at Austin.

Wimberly M C, Spies T A, Long C J, et al. 2000. Simulating historical variability in the amount of old forests in the Oregon Coast Range[J]. Conservation Biology, 14(1): 167-180.

Wu J G, Loucks O L. 1995. From balance of nature to hierarchical patch dynamics: A paradigm shift in ecology[J]. The Quarterly Review of Biology, 70(4): 439-466.

Xi W M, Coulson R N, Birt A G, et al. 2009. Review of forest landscape models: Types, methods, development and applications[J]. Acta Ecologica Sinica, 29(1): 69-78.

Xi W M, Coulson R N, Waldron J D, et al. 2008. Landscape modeling for forest restoration planning and assessment: Lessons from the southern Appalachian Mountains[J]. Journal of Forestry, 106(4): 191-197.

Xi W M, Waldron J D, Coulson R N, et al. 2007. Landscape modeling for forest restoration: Concepts and applications $[\mathrm{C}] / /$ Stanturf J. Proceedings of the IUFRO conference on forest landscape restoration. Seoul, Korea: Korea Forest Research Institute: 268.

Yang J, He H S, Gustafson E J. 2004. A hierarchical fire frequency model to simulate temporal patterns of fire regimes in LANDIS[J]. Ecological Modelling, 180(1): 119133.

Yang J, He H S, Shifley S R, et al. 2007. Spatial patterns of modern period human-caused fire occurrence in the Missouri Ozark Highlands[J]. Forest Science, 53(1): 1-15.

Yemshanov D, Perera A H. 2002. A spatially explicit stochastic model to simulate boreal forest cover transitions: General structure and properties[J]. Ecological Modelling, 150 (1): 189-209.

\title{
Advances in forest landscape modeling: Current research and applications
}

\author{
XI Weimin ${ }^{1,2}$, DAI Erfu' ${ }^{3}$, HE Hongshi ${ }^{4,5}$ \\ (1. Department of Biological and Health Sciences, Texas A\&M University, Kingsville TX 78363, USA; \\ 2. Institute of Applied Ecology, CAS, Shenyang 110016, China; 3. Key Laboratory of Land Surface Pattern and \\ Simulation, Institute of Geographic Sciences and Natural Resources Research, CAS, Beijing 100101, China; \\ 4. Northeast Normal University, Changchun 130024, China; 5. School of Natural Resources, University of \\ Missouri, Columbia MO 65211, USA)
}

\begin{abstract}
Forest landscape models simulate temporal change of forests using spatially referenced data across a broad spatial scale (landscape scale) generally larger than a single forest stand. Spatial interactions between forest stands are a key component of such models. These models can incorporate other spatiotemporal processes such as natural disturbances (e.g., wildfires, hurricanes, outbreaks of native and exotic invasive pests and diseases) and human influences (e.g., harvesting and commercial thinning, planting, fire suppression). The models are increasingly used as tools for studying forest management, ecological assessment, restoration planning, and examining the impact of climate change. In this article, we define forest landscape models and discuss their development, components, and types. We also review commonly used methods and approaches in modeling, their applications, and the strengths and limitations of different forest landscape models. New developments in computer sciences, geographic information systems (GIS), remote sensing technologies, decision-support systems, and geo-spatial statistics have provided opportunities for developing new generations of forest landscape models that are more valuable in ecological research, restoration planning, and resource management.
\end{abstract}

Key words: forest landscape change; landscape models; temporal and spatial scales; forest management and planning; resource conservation and restoration; decision-making models 